\title{
Armonizando trabajo y familia en Bogotá-Colombia: la conexión doméstica
}

\section{Harmonizing work and family in Bogota-Colombia: the domestic connection}

\author{
Milagrosa Hernáez García* \\ Sandra Idrovo Carlier**
}

\section{RESUMEN}

Esta investigación busca conocer cómo el ámbito familiar, específicamente el trabajo doméstico, afecta el ámbito laboral, determinando si tiene importancia al conciliar Trabajo - Familia (TF). Es una investigación exploratoria, descriptiva y cualitativa, en la que se emplea la estratificación ya existente en Bogotá y que hace referencia a los niveles socioeconómicos (utilizando los estratos más altos, 5 y 6) aplicándola a varones y mujeres mayores de 40 años que estuvieran trabajando. Los resultados indican que el género es un factor determinante al asumir la responsabilidad del trabajo doméstico, y éste es importante tanto para varones como mujeres al tratar de conciliar TF.

Palabras clave: trabajo, familia, conciliación, trabajo doméstico, Colombia

\begin{abstract}
This research looks at the work family interface in Colombia focusing on how the family domain, specifically household work, affects work and therefore determining if it has any relevance in the harmonizing of W-F. It is exploratory, descriptive and mainly qualitative. A survey was handed to a pre-established socio-economical stratified sample of Bogota's upper class population (5-6), men and women who were 40 years or older, and working. Results indicate that for men and women, household work plays an important role in balancing W-F. They also show a rise in men's involvement with domestic chores, with gender still being a determinant factor of who assumes responsibility.
\end{abstract}

Keywords: work, family, harmonizing, domestic, Colombia

* Española, Psicóloga, Investigadora de INALDE - Escuela de Dirección y Negocios de la Universidad de la Sabana, Colombia. Correspondencia con la autora: milahernaez@yahoo.es.

** Ecuatoriana, Periodista, M.A. Ciencias de la Información, Ph.D. Comunicación, Directora de Investigación de INALDE - Escuela de Dirección y Negocios de la Universidad de La Sabana, Colombia. Correspondencia con la autora: sandra.idrovo@inalde.edu.co 
El trabajo y la familia son los ámbitos vitales más importantes para la mayoría de los adultos. Y aun cuando determinar cuál de ellos es el más importante varía entre las diferentes culturas, la afirmación es válida para todas ellas. Las intersecciones de ambos planos han sido el objeto de un considerable cuerpo de literatura en los últimos ańos (Barling \& Sorensen, 1997; Greenhaus \& Parasuraman, 1999). Estos estudios han aparecido en revistas de diferentes áreas: psicología aplicada, estudios familiares, relaciones industriales y trabajo, administración, negocios, sociología, demografía, psicología del desarrollo, estudios de la mujer, trabajo social, etc. (Drago \& Kashian, 2003).

La mayoría, si no todas, las investigaciones sobre la interfaz trabajo-familia corresponden a países desarrollados, principalmente los Estados Unidos, subrayando la falta de miradas provenientes de otras culturas y naciones. Ciertamente hay excepciones y se encuentran trabajos en los que diferentes culturas han sido estudiadas (Spector et al., 2004) o en los que se han hecho comparaciones internacionales (Shaffer et al., 2005; Westman, 2005). De todas formas, la gran mayoría de las investigaciones han sido hechas en inglés y dentro de marcos específicos que tienen categorías de análisis que no son aplicables a otras culturas o que tienen diferente evaluación cultural. Las investigaciones de los países de América Latina en el área de trabajo-familia son escasas (Poelmans et al., 2005). Se pueden encontrar algunos datos aislados de países específicos tales como México, Brasil y Colombia en la poca literatura cross - cultural que existe (Spector et al., 2005; Shaffer et al., 2005). Tal y como indica Poelmans (2005), es tan sólo recientemente que con el aumento de la participación femenina en el mercado laboral, la transición a familias con doble ingreso, y los cambios socioeconómicos generales de América Latina, que los conflictos trabajo - familia han empezado a llamar la atención de organizaciones nacionales e internacionales, y de los investigadores, debido especialmente a la importancia que la familia como institución todavía tiene en la cultura latinoamericana.

El objetivo general de la presente investigación es comenzar a analizar la interfaz trabajo-familia en Bogotá - Colombia, centrándose en cómo el ámbito de la familia, especialmente el trabajo del hogar, afecta el ámbito del trabajo y, por ende, determinar si tiene alguna relevancia en la armonización de trabajo y familia.

\section{Trabajo y familia: ¿Aliados o enemigos?}

Las intersecciones entre trabajo y familia han sido estudiadas desde diferentes puntos de vista, pero parece existir una perspectiva de conflicto predominante en la literatura de trabajo - familia que subraya el estrés y la afectación del bienestar (Greenhaus \& Powell, 2006). La lógica detrás de la perspectiva del conflicto sostiene que dados una cantidad limitada de tiempo y energía humana, los individuos que toman parte en múltiples roles, inevitablemente experimentan conflictos y estrés que afectan su calidad de vida. Los investigadores del conflicto trabajo - familia han identificado una naturaleza bidi- 
reccional (TIF y FIT ${ }^{1}$ ) (por ej. Adams et al., 1996; Duxbury et al., 1992; Frone et al., 1992; Gutek et al., 1991; Thomas \& Gansters, 1995) y facetas multi-dimensionales del modelo (basadas en el tiempo, la tensión y el comportamiento) (Greenhaus \& Beutell, 1985). Desde entonces Carlson, Kacmar y Williams (2000) han refinado la medición de TIF y FIT en tres dimensiones cada uno, con subtipos de conflicto basado en el tiempo, conflicto basado en la tensión y conflicto basado en el comportamiento. Lo que muestra la literatura es una permeabilidad de las fronteras de los ámbitos del trabajo y la familia que, sin embargo, requieren más investigación sobre otros mecanismos explicativos y moderadores de la relación entre ámbitos (Ford et al., 2007).

$\mathrm{Al}$ mismo tiempo, parece haber un creciente reconocimiento entre investigadores de las interdependencias positivas entre el trabajo y la familia, y términos tales como enriquecimiento (Greenhaus \& Powell, 2006; Kirchmeyer, 1992; Rothbard, 2001), propagación positiva (Crouter, 1984; Grzywacz, 2000; Grzywacz, Almeida, \& McDonald, 2002; Grzywacz \& Marks, 2000a,b; Hammer et al., 2002; Hanson, Colton \& Hammer, 2003; Kirchmeyer, 1992b, 1993, 1995; Stephens, Franks \& Atienza, 1997; Sumer \& Knight, 2001; Voydanoff, 2001), acrecentamiento (Ruderman, Ohlott, Panzer \& King, 2002; Tiedje et al., 1990) y facilitación (Frone, 2003; Tompson \& Werner, 1997; Wayne, Musisca \& Fleeson, 2004) han surgido. Dentro de este giro positivo, y también en trabajos previos, la influencia familia - trabajo ha sido la que ha recibido menos atención (Crouter, 1984), aun cuando las investigaciones adelantadas muestran su importancia en la comprensión plena de la interfaz TF (Frone et al., 1997, 2003; Greenberger \& O’Neil, 1990; Gutek et al., 1991; Kinnunen \& Gerris, 1996; Spitze, 1988).

Lo que la literatura también muestra es que para cada ámbito de la interfaz TF parece haber variables específicas que interfieren con el otro ámbito (Frone et al, 1997; Greenhaus \& Beutell, 1985) y que éstas estaban negativamente relacionadas con resultados específicos de cada ámbito: FIT está negativamente relacionado con el desempeño en el trabajo, mientras que TIF está negativamente relacionado con el desempeño familiar (Frone et al., 1997; Lingard \& Francis, 2007). En otras palabras, se encontró que el trabajo afecta la vida familiar (y viceversa) mediante la experiencia subjetiva del conflicto trabajo - familia. Viéndolo desde otra perspectiva, pero en la misma línea, Ford et al. (2007) concluyeron que una cantidad importante de la satisfacción en los ámbitos de la familia y del trabajo se refiere a estresores y soportes que están fuera de estos ámbitos.

El trabajo doméstico, entendido como tareas del hogar y obligaciones de cuidado de nińos, está dentro de las variables que pueden causar interferencia del dominio familiar, sea en forma de presión por tiempo (por ej.: el tiempo dedicado al trabajo doméstico o

1 TIF=Trabajo interfiere en la familia, FIT= Familia interfiere en el trabajo. 
al cuidado de los nińos interfiere con el tiempo del trabajo) (Baltes \& Heydens-Gahir, 2003; Frone et al., 1992a) o problemas basados en la tensión (por ej.: la presencia de los niños ha sido identificada con un sentimiento de pérdida de control sobre el balance trabajo - familia, Batt \& Valcour, 2003; Tausig \& Fenwick, 2001). La división del trabajo doméstico es central, por ejemplo, para entender cómo las parejas equilibran el trabajo y la vida familiar (Zimmerman et al., 2003), pero los hallazgos relativos al trabajo doméstico y a la interfaz trabajo - familia son diversos. Voydanoff (1988) encontró que el tiempo de trabajo doméstico no se asociaba con el conflicto trabajo - familia. Sin embargo, por lo menos un estudio ha encontrado que la responsabilidad del trabajo doméstico estaba positivamente relacionada con el conflicto trabajo - familia (Jansen, Kant, Kristensen \& Nijhuis, 2003). Algunas de las inconsistencias en la literatura pueden deberse a la diferencia en la definición de trabajo doméstico. Ciertos académicos han usado definiciones de trabajo doméstico que se refieren tan sólo a las labores del hogar (por ej.: cocinar, limpiar, mantenimiento, jardinería) (Caplan \& Shooler, 2006), separándolo del cuidado de los nińos o por lo menos de los factores psicológicos incluidos en el cuidado de los niños o de cualquier otra persona (Noonan et al., 2007). Otros han usado una definición extendida de trabajo doméstico que incluye el trabajo en el hogar y variables como la carga emocional y el acrecentamiento o no de estatus asociados a ese trabajo. Estos académicos argumentan que, al igual que otras formas de trabajo doméstico, también requieren de energía y trabajo por parte de quienes la realizan (Coverman, 1989; Stevens et al., 2007).

Otros investigadores sostienen que la división de trabajo doméstico es relevante para las relaciones sólo cuando las preferencias personales son consideradas (Blair \& Johnson, 1992; Kluwer \& Heesink, 1996). Por ejemplo, estudios han mostrado que la satisfacción con la división del trabajo a menudo es un mejor indicador de la satisfacción conyugal y de la cohesión familiar que la cantidad real de trabajo realizado (Stevens, Kiger \& Mannon, 2005; Stevens, Kiger \& Riley, 2001, 2002) o el tiempo empleado en ello. De acuerdo con la literatura, hay tres perspectivas teóricas principales para predecir el tiempo usado en los trabajos del hogar: disponibilidad de tiempo (el trabajo del hogar se asigna de acuerdo con el personal disponible en el hogar y en relación con la cantidad de trabajo a ser realizado), recursos relativos (ingreso, educación, ocupación o cualquier otro recurso traído a la relación determina la cantidad de trabajo del hogar que el individuo hace a través de procesos de poder o de maximización de eficiencia) y el género (el trabajo del hogar sería una extensión simbólica de las relaciones entre los géneros) (revisado en Bianchi et al., 2000).

Sea que las nuevas circunstancias sociales y económicas están empujando a los hombres a involucrarse con las labores del hogar, o no, las investigaciones parecen indicar que las mujeres siguen siendo las principales responsables del trabajo del hogar y el cuidado de las familias. Por ejemplo, ellas hacen considerablemente más trabajo doméstico y de cuidado de los niños que los hombres y, obviamente, invierten más tiempo en ello 
(Barnett \& Hyde, 2001; Hochschild, 1999; Pocock, 2003; Rothausen, 2003), sin tomar en cuenta que también pueden tener un trabajo de tiempo completo. Esta situación hace que las mujeres sean especialmente vulnerables a verse afectadas por lo doméstico en sus esfuerzos de balancear trabajo y familia. Lilly et al. (2006) encontraron que hay claras diferencias entre las percepciones de hombres y mujeres respecto de la interferencia de la familia con el trabajo. Las mujeres, en su mayor parte, se ven más afectadas por las obligaciones familiares que los hombres. Este hallazgo, dicen, tiene repercusiones potenciales en el sitio de trabajo que pueden incluir un menor desempeño laboral de las mujeres, sueldos más bajos para ellas y mayor rotación.

\section{Trabajo y Familia en Bogotá-Colombia}

La armonización del trabajo y la vida familiar no es fácil para los bogotanos. Por un lado, la ley establece una semana laboral de 48 horas que, combinada con largas distancias y un sistema lento de transporte público, reduce el tiempo diario y semanal disponible. Por otro lado, la situación económica del país pone un peso muy grande sobre las familias que necesitan ingreso extra para cubrir un poco más que las necesidades básicas. Al igual que en el resto de América Latina, está aumentando el número de hogares con doble ingreso: para el 2002, el porcentaje de hogares con dos ingresos subió del 27\% al 33\% (Comisión Económica para América Latina [CEPAL], 2004).

Los cambios son evidentes a nivel de la estructura familiar. El Siglo XXI encontró una familia urbana latinoamericana bastante diferente. Los hogares nucleares y de dos padres han decrecido. $Y$ aun cuando la familia nuclear sigue prevaleciendo, el modelo tradicional de familia con un padre que provee, una madre ama de casa y niños corresponde tan solo al 35\% de los hogares; en otras palabras, ya no es la mayoría de los hogares en América Latina. Para el 2002, un número cada vez mayor de hogares tienen mujeres solas cabeza de familia, incluyendo la familia extendida que sigue siendo el $14 \%$ de los hogares. Para Colombia la familia extendida representa un 24,8\% de los hogares y la familia nuclear, de padre y madre con nińos representa el $40,1 \%$ de las familias (CEPAL, 2004).

De acuerdo con la CEPAL, estos cambios se asocian, entre otras circunstancias, con el aumento del trabajo femenino pago: para el 2002, las mujeres representaban el 49,7\% de la fuerza laboral en las áreas urbanas en América Latina. En Colombia las mujeres son el $51 \%$ de la población y representan el $48 \%$ de la fuerza laboral en las áreas urbanas (Departamento Administrativo Nacional de Estadística [DANE], 2005). Colombia también es uno de los países latinoamericanos ${ }^{2}$ con las cifras más altas $(10,7 \%)$ de hogares nucleares en los que la cabeza es una mujer (CEPAL, 2004).

2 Los otros son: Bolivia, Costa Rica, Honduras, Panamá y la República Dominicana. 
Pero las dificultades para equilibrar trabajo y familia surgen no solo por condiciones externas y estructurales. También hay razones culturales. Los colombianos le dan un gran valor a la familia, valores que se concretan en elecciones en sus vidas personales y laborales. La Encuesta Nacional de Valores ${ }^{3}$ (ENV, 2006) indicaba que para el 98,70\% de los colombianos la familia es 'Muy Importante'; los progenitores deben ser amados y respetados sin importar sus méritos $(90,61 \%)$ y ellos a su vez deben hacer toda clase de sacrificios (incluyendo sacrificios personales) por el bienestar de sus hijos $(87,16 \%)$. Sin importar género, región, ni condición económica, los colombianos aprecian a la familia. Pero ellos también valoran el trabajo: para al menos el 93,26\% de ellos, el trabajo es una parte 'muy importante' de sus vidas. La encuesta muestra que el $51 \%$ de los colombianos considera que el trabajo, incluyendo el trabajo realizado en el hogar y / o en la escuela, hace que la vida valga la pena (frente al tiempo libre) y por lo menos el $30 \%$ de los colombianos de distintas edades y regiones considera que el trabajo siempre debe ir primero, aun si esto implica recortar el tiempo reservado para otras cosas. Un $25 \%$ considera que no es ni el trabajo ni el tiempo libre, sino un balance de los dos, lo que hace que la vida valga la pena. ¿Por qué le dan tanto valor al trabajo? Porque consideran que el trabajo es, en primer lugar, un deber para con la sociedad (79\%) $\mathrm{y}$, en segundo, una fuente de crecimiento personal. Y aun cuando los porcentajes de aquellos que prefieren el trabajo son menores a los que prefieren la familia, la fortaleza de las preferencias presenta un escenario en el cual el conflicto entre los dos ámbitos tiene muchas posibilidades de generarse.

Dentro de ese escenario, los resultados obtenidos por Spector et al. (2005) respecto de la relación entre estrés en la relación trabajo-familia y tensión ocupacional pueden ser explicados mejor. El estudio de Spector exploraba las relaciones trabajo - familia con otras variables que se ha encontrado que estaban relacionadas con la presión, ya sea como antecedentes potenciales (horas de trabajo, número de nińos) o consecuencias (satisfacción laboral, tensión mental y tensión física). Lo hicieron a lo largo de una muestra diversa de países, incluyendo a Colombia. El propósito era ver en qué medida se podía hacer una generalización sobre las mediciones obtenidas en otros países. El resultado mostró una importante correlación de horas de trabajo y número de niños, y presión en la relación trabajo - familia para los países anglosajones (Estados Unidos, Reino Unido y Australia), pero para los demás había considerables discrepancias. Para Colombia, que reportó el número más alto de horas de trabajo, no había correlación del trabajo como antecedente de presión trabajo - familia. Colombia, junto con México, reportó el más alto nivel de satisfacción y nuevamente ninguna relación con la presión trabajo - familia. Quizás a la luz de la alta valoración que los colombianos otorgan al

3 La encuesta se realizó en el 2004. Los resultados fueron publicados en el 2006. La muestra garantiza una validación nacional para los resultados siguiendo los parámetros del World Value Survey. 
trabajo y mencionada anteriormente (ENV, 2006) estos resultados se entiendan mejor. Pero, como Spector et al. (2004) sugieren, las mediciones trabajo - familia usadas fueron desarrolladas dentro de un contexto occidental, de forma que las particularidades culturales de la relación trabajo - familia de países no occidentales siguen ocultas. De ahí el cuestionamiento de la validez de las medidas a ser usadas en los distintos países.

Las especificidades culturales encierran la forma en la que las familias organizan sus rutinas y prácticas y lo que se espera de cada uno de los miembros del hogar. Para los colombianos, el cuidado de la familia y las labores del hogar son principalmente el dominio de la mujer (León, 1989). En el último Censo Nacional (DANE, 2005), el $30,1 \%$ de las mujeres reportaron haber hecho algún tipo de labores domésticas durante la semana anterior frente al 2,1\% de hombres. Pero al mismo tiempo las mujeres son la cabeza del 30\% de los hogares colombianos y la principal fuente de ingreso para esas familias. Esto ofrece una perspectiva bastante difícil para las mujeres que tratan de balancear el trabajo y la familia. En un reporte reciente de las mujeres en las corporaciones de América Latina, las ejecutivas colombianas mencionaban su negativa a seguir subiendo la escalera corporativa porque eso pondría en riesgo la estabilidad de su vida familiar. Sin importar si eran casadas o solteras, ellas explícitamente expresaban sus dificultades para distribuir su tiempo entre trabajo y familia (Maxfield, 2005) ${ }^{4}$. Las decisiones tomadas por estas mujeres parecerían consistentes con la aseveración mencionada anteriormente, que los colombianos creían que los padres deben sacrificarse por el bienestar de sus hijos (ENV, 2006). Y ellos, los padres, están contentos de hacerlo; no es una causa de infelicidad. Un dato importante producido por la ENV respecto de las mujeres es precisamente la razón de su infelicidad. La encuesta mostró que es más probable que las mujeres colombianas se sientan infelices a que los hombres colombianos lo hagan, y seńalaron que las principales causas para ello eran los problemas de salud y la poca satisfacción con la situación económica. Por otro lado, que la falta de control sobre sus vidas y la libertad de escoger (empowerment) no estaban significativamente ligadas a la infelicidad; la afiliación religiosa y el estado conyugal sí lo estaban. Para las mujeres colombianas el estar casadas reduce en 36\% la posibilidad de reportar infelicidad, mientras que la separación aumenta esa posibilidad en $87 \%$.

Otra de esas especificidades culturales que uno debe considerar es el nivel al cual un conflicto llega a la arena pública. En este caso, para la interfaz trabajo - familia es importante mirar cómo las organizaciones, públicas y privadas, se enfrentan a la situación. En Colombia los resultados del IFREI 2005 (Idrovo, 2006) mostraron que aun cuando los ejecutivos son conscientes de la importancia del balance trabajo - familia,

4 El reporte también indica cómo las mujeres latinoamericanas ocupan un 25-35\% de las posiciones gerenciales y Colombia, junto con México, está en la cabeza de la lista de los países en los cuales la presencia femenina en la alta gerencia es evidente.

5 IESE Family Responsible Employer Index 
en la realidad, las estrategias no reflejan esta preocupación toda vez que el 60\% de las compañías declaran que la implementación de políticas orientadas a la resolución de estos problemas ni siquiera hace parte de la agenda de las empresas.

Esto es evidente en los bajos porcentajes de implementación de políticas (por ejemplo, políticas de servicio para el cuidado de familiares: centros de cuidado diurno para niños, centros de atención para ancianos, etc.) o en el caso de políticas de entrenamiento y desarrollo; el bajo porcentaje de compañías que promueven la educación de sus empleados como padres o esposos, capacitación del balance trabajo - familia, comparado con el soporte dado al entrenamiento sobre cómo manejar el estrés o el tiempo. Facilitadores FR también reflejan la ausencia de políticas responsables con la familia en la agenda de los ejecutivos; en el $42 \%$ de las compañías sólo muy pocos ejecutivos dan un buen ejemplo de buscar un buen balance trabajo - familia. Esto se puede explicar por el alto valor que los colombianos le dan al trabajo y por la percepción de que el trabajo va primero, sacrificando el tiempo dedicado a otras cosas. Pero también plantea la cuestión de si la dedicación de tiempo y trabajo a la familia es la medida adecuada para la interfaz trabajo - familia. Ejemplos de ejecutivos que buscan un buen balance trabajo - familia usualmente se comparan contra la cantidad de tiempo dedicada al trabajo o con la familia y / o en actividades familiares.

$\mathrm{Y}$ esto es importante porque la alta apreciación de los colombianos por la familia es evidente en la cultura de las companías. Los resultados del IFREI 2005 también mostraron que la presencia de obstáculos que imposibiliten una conciliación entre el trabajo y la familia no juegan un papel vital. Por el contrario, algunos determinantes familiares indican una fuerte presencia: por ej. el $71 \%$ de las compañías establecen que muchas veces o siempre el hablar de la familia en el trabajo está bien visto; y el $57 \%$ respondió que los ejecutivos a lo largo de la compañía comparten y apoyan -fuertemente o siempre- la idea de que la familia debe ser tomada en cuenta al asignar responsabilidades. Esto se podría interpretar como una posibilidad que facilitaría la implementación de las políticas de equilibrio trabajo - familia, y una vez se ha asumido que su presencia es necesaria, volverse tangible en la estrategia general de la compañía (Idrovo, 2006).

\section{El presente estudio}

Lo que hemos dicho hasta ahora genera varias preguntas: ¿Cómo armonizan los colombianos el trabajo y la familia, considerando que ambos son altamente valorados? ¿Cómo manejan el alto número de horas de trabajo y el bajo porcentaje de implementación de políticas responsables para con la familia, con la dedicación a la familia? ¿Qué significa dedicación a la familia: hacer cosas por otros miembros, encargarse de las tareas domésticas, dedicar tiempo, apoyo emocional, escuchar? ¿Cómo hacen las mujeres considerando que trabajan adentro y afuera del hogar? ¿Qué mecanismos y / o rutinas les ayudan, tanto a hombres como a mujeres, a armonizar la familia y el trabajo? ¿Qué 
papel cumple el trabajo doméstico en el balance del trabajo y la familia? Éstas fueron algunas de las preguntas que orientaron el trabajo de investigación.

Ya que hay escasa o ninguna literatura disponible sobre la interfaz trabajo - familia, al menos en las revistas colombianas y latinoamericanas, se hicieron algunas presunciones con información tomada de investigaciones hechas en otros países y comparando y contrastando con los puntos esbozados anteriormente.

La primera decisión tomada fue enfocarse en la dimensión familia-trabajo de la interfaz TF. La lógica detrás de esto fue comenzar a trabajar con lo que parecía ser tan altamente valorado por los colombianos (ENV, 2006); de alguna manera no había sido tomado en cuenta por la literatura (Crouter, 1984) y podía dar algunas sorpresas ya que como Greenhaus \& Powell (2006: 76) señalaron: "respecto de la direccionalidad del enriquecimiento, nueve de los once estudios que evaluaban ambas direcciones de enriquecimiento hallaron que el enriquecimiento de familia a trabajo era sustancialmente más fuerte que el enriquecimiento de trabajo a familia."

La segunda decisión era escoger el trabajo doméstico como el estresor del ámbito familiar cuya incidencia iba a ser estudiada. Las razones eran: La similitud en que las mujeres asumen la mayoría de las tareas domésticas en los diferentes países; el hecho de que compartir el trabajo del hogar es importante en las relaciones familiares (Stevens et al., 2005; 2001; 2002) así como una posible fuente de conflicto familia - trabajo; y la naturaleza del trabajo doméstico mismo: tan vital y al mismo tiempo con tan baja consideración social y económica. Para este estudio, trabajo doméstico se definió como el conjunto de actividades que cada hogar requiere y que los miembros del núcleo familiar esperan en su espacio físico.

También se decidió no medir el tiempo gastado en labores domésticas (horas) sino usar una categoría cualitativa: asumir responsabilidad sobre tales tareas. Nuevamente las razones vinieron de las características especiales de lo doméstico, donde el hacer las tareas puede ser realizado por otra persona (empleados domésticos, otros miembros de la familia), pero la responsabilidad del resultado sigue recayendo sobre uno de los miembros del hogar o, por lo menos, la responsabilidad se experimenta y / o se cede como tal, por los miembros de la familia. Y esa responsabilidad, o la percepción de ser responsable, es lo que determina patrones y rutinas que se pueden traslapar con el ámbito del trabajo.

Aun cuando la investigación era exploratoria, descriptiva y principalmente cualitativa, se plantearon algunas hipótesis:

$\mathrm{H}_{1:}$ El género, el estado civil y las personas con quienes se vive están asociados con la forma en la cual se ejercen las responsabilidades sobre los trabajos del hogar.

$\mathrm{H}_{2}$ : Es más probable que las mujeres (a) que asumen la responsabilidad de las tareas del 
hogar antes que los hombres (b) que también asumen responsabilidades, consideren que el trabajo doméstico es importante en el balance entre familia y trabajo.

$\mathrm{H}_{3:}$ El estado civil y las personas con quienes se vive están asociadas con la posibilidad de considerar que el trabajo doméstico es importante en el balance entre familia y trabajo.

$\mathrm{H}_{4}$ : Es más probable que quienes están insatisfechos con la forma como se desempeñan las tareas domésticas encuentren que el trabajo doméstico es importante en el balance entre familia y trabajo.

Se decidió comenzar esta investigación exploratoria con las áreas urbanas, en este caso Bogotá, y con una estratificación preestablecida: la estratificación socioeconómica ${ }^{6}$. Se tomaron los dos estratos más altos (5 media alta- 6 alta), de 40 años de edad o más, que se encontraran trabajando y que fueran proporcionalmente hombres y mujeres semejantes a la distribución nacional. Teniendo en cuenta que el total de personas en estratos 5 y 6 en la ciudad es de 343.393 (2002) y considerando que a nivel nacional la distribución de varones y mujeres es del $49 \%$ y $51 \%$ respectivamente, y la de personas mayores de 40 ańos es de 20,5\%, se llegó a una muestra representativa de 138 , considerando un nivel de confianza del 95\% (1,96) y de error muestral del 5\%. La recolección de datos se realizó entre noviembre 2005 y mayo 2006. Los cuestionarios fueron entregados a personas mayores de 40 años de edad, de los cuales se entregaron el $51 \%$ a mujeres y el $49 \%$ restante a los hombres. Del total se eliminaron 3 por estar incompletas, quedando un total de 135.

El cuestionario fue diseñado de acuerdo con las decisiones anteriormente mencionadas, y usando preguntas abiertas y cerradas. Se compone de 4 partes: 1) Datos demográficos: género, edad, lugar de nacimiento, profesión, ocupación, educación, estado civil y condiciones del diario vivir; 2) Definición de labores domésticas: si se consideran trabajo o no, y qué clase de tareas forman parte de la definición de labores domésticas; 3) Qué clase de responsabilidad se asume sobre las labores domésticas y cómo se cumple con tal responsabilidad; quién hace qué más frecuentemente y si el encuestado(a) está satisfecho(a) o no con el trabajo doméstico en su casa, y finalmente la relación entre el trabajo doméstico en la armonía entre trabajo y familia; 4) La cuarta parte consiste en preguntas relacionadas con las cualificaciones del posible servicio doméstico pago.

Para los fines del presente estudio nos centraremos en la parte 3) de la encuesta, que corresponde a las preguntas 4, 4.1, 4.2.1, 4.2.2, 4.2.3, 4.2.4; 5.1, 5.2, 5.3, 5.4, 5.8; $6.1,6.2,6.3,6.4,6.8 ; 7$.

6 La población en Colombia se encuentra dividida en estratos que van del 1 al 6, donde el 1 es el estrato socioeconómico más bajo y el 6 es el más alto. Los estratos 5 y 6 son aquellos que ganan salarios equivalentes a 8 o más salarios mínimos mensuales vigentes; corresponden al 5,2\% de la población de Bogotá (DAPD, Área de Estadística y Monitoreo, 2002). 


\section{Tabla I}

\begin{tabular}{|c|c|}
\hline & Preguntas $4-7$ \\
\hline \multirow{7}{*}{ 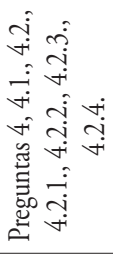 } & 4. ¿Asume Usted alguna responsabilidad sobre las labores del hogar? \\
\hline & 4.1. ¿Sobre qué? \\
\hline & 4.2. ¿Cómo cumple Usted con esa responsabilidad? \\
\hline & 4.2.1. Haciendo mis tareas cuando vuelvo a casa después del trabajo \\
\hline & 4.2.2. Tratando de arreglar las cosas desde el trabajo \\
\hline & 4.2.3. Solicitando flexibilidad en el trabajo de forma que pueda realizar \\
\hline & 4.2.4. Pidiendo la ayuda de quienes viven conmigo \\
\hline \multirow{6}{*}{ 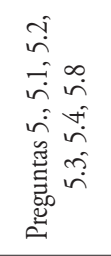 } & $\begin{array}{l}\text { 5. En su familia, ¿quién hace las siguientes tareas más frecuentemente? Opciones: Yo mismo(a), } \\
\text { mi cónyuge, otros (hijos, padres), trabajadores del servicio doméstico. }\end{array}$ \\
\hline & 5.1. Cocinar \\
\hline & 5.2. Limpiar \\
\hline & 5.3. Lavar la ropa \\
\hline & 5.4. Mantenimiento \\
\hline & 5.8. Cuidar a los niños \\
\hline \multirow{6}{*}{ 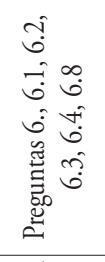 } & $\begin{array}{l}\text { 6. ¿Se encuentra Usted satisfecho con la forma con la que se hacen las labores domésticas? } \\
\text { Sí o No }\end{array}$ \\
\hline & 6.1. Cocinar \\
\hline & 6.2. Limpiar \\
\hline & 6.3. Lavar la ropa \\
\hline & 6.4. Mantenimiento \\
\hline & 6.8. Cuidar de los niños \\
\hline 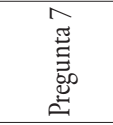 & 7. ¿Cree Usted que las labores domésticas son importantes para el balance trabajo y familia? \\
\hline
\end{tabular}

Las preguntas de esta sección eran cerradas.

\section{Resultados}

Tabla II. Chi2 con relación a género, estado civil y con quién vive

\begin{tabular}{|c|c|c|c|}
\hline No. Pregunta & gen & ec & $\mathrm{cv}$ \\
\hline 4 & $0,004^{*}$ & 0,155 & -...-..- \\
\hline 4.1 & 0,595 & 0,948 & -..--- \\
\hline 4.2.1. & 0,631 & 0,095 & 0,299 \\
\hline 4.2.2. & 0,721 & 0,576 & -..--.- \\
\hline 4.2.3. & -..-..-- & -....... & -.....-. \\
\hline 4.2.4. & 0,345 & 0,218 & $0,025^{*}$ \\
\hline 5.1 & $0,019^{*}$ & $0,0006^{*}$ & --.---- \\
\hline 5.2. & $0,005^{*}$ & 0,014 & -..-..- \\
\hline 5.3. & 0,117 & -...-.- & -.-.--- \\
\hline 5.4 & $0,004^{*}$ & -.-.-.- & -.--.-- \\
\hline 5.8. & 0,78 & $0,002^{*}$ & -...-.- \\
\hline 6.1 & 0,057 & 0,778 & -.--.-- \\
\hline 6.2 & 0,57 & 0,817 & -.---.- \\
\hline 6.3 & 0,742 & 0,858 & 0,988 \\
\hline 6.4 & 0,205 & -......- & -...-..- \\
\hline 6.8 & 0,195 & $0,007^{*}$ & $0,0009^{*}$ \\
\hline 7 & 0,636 & 0,969 & -.....- \\
\hline $\begin{array}{l}\mathrm{p}^{*}<0,05 \\
\text { gen=género } \\
\text { ec=estado civil } \\
\mathrm{cv}=\text { con quién vive }\end{array}$ & & & \\
\hline
\end{tabular}




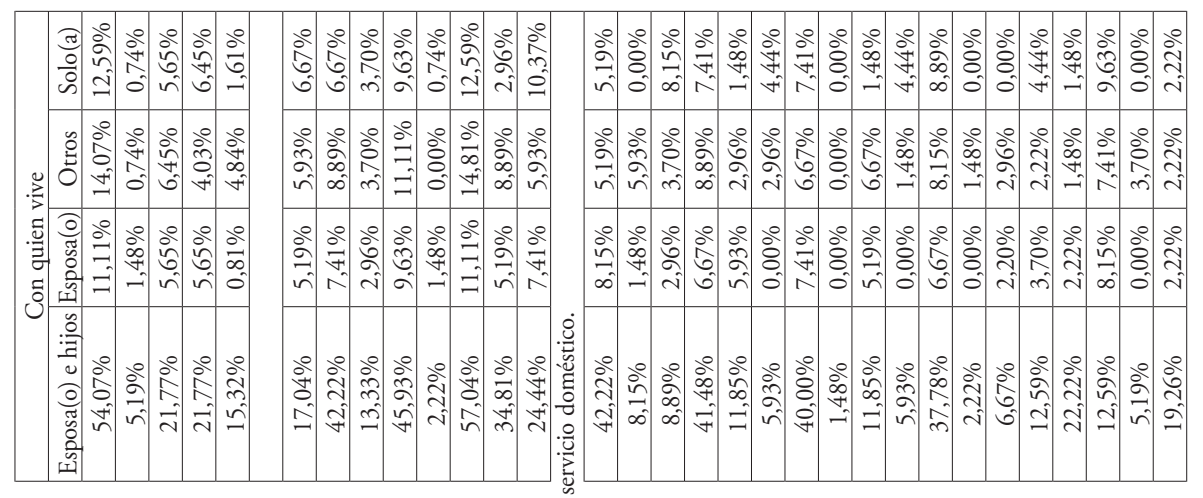

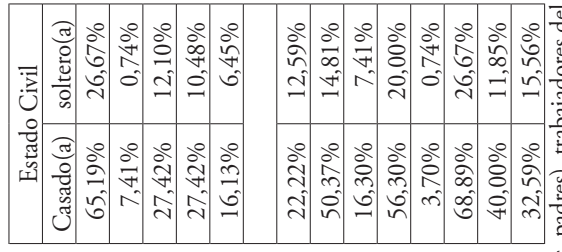

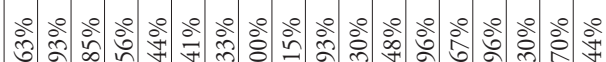
नी जी $=$ जी

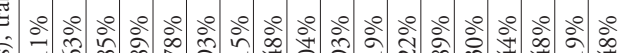

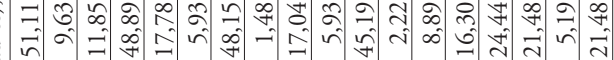

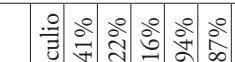

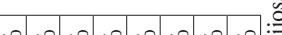

(3)

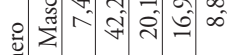

ले लेच బิ

1

نั

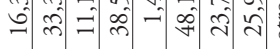

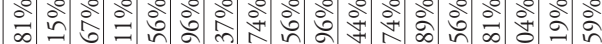

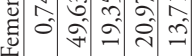

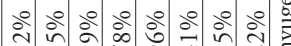

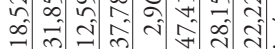

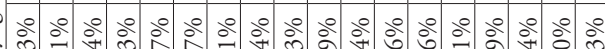

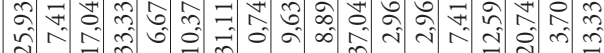

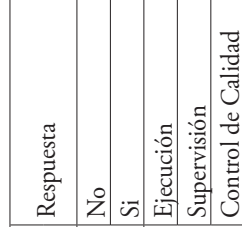

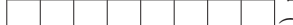

:

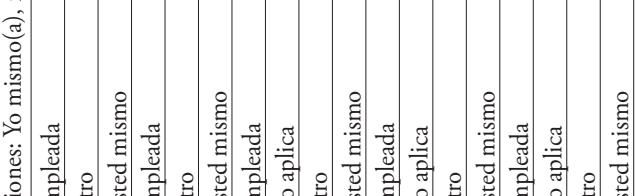

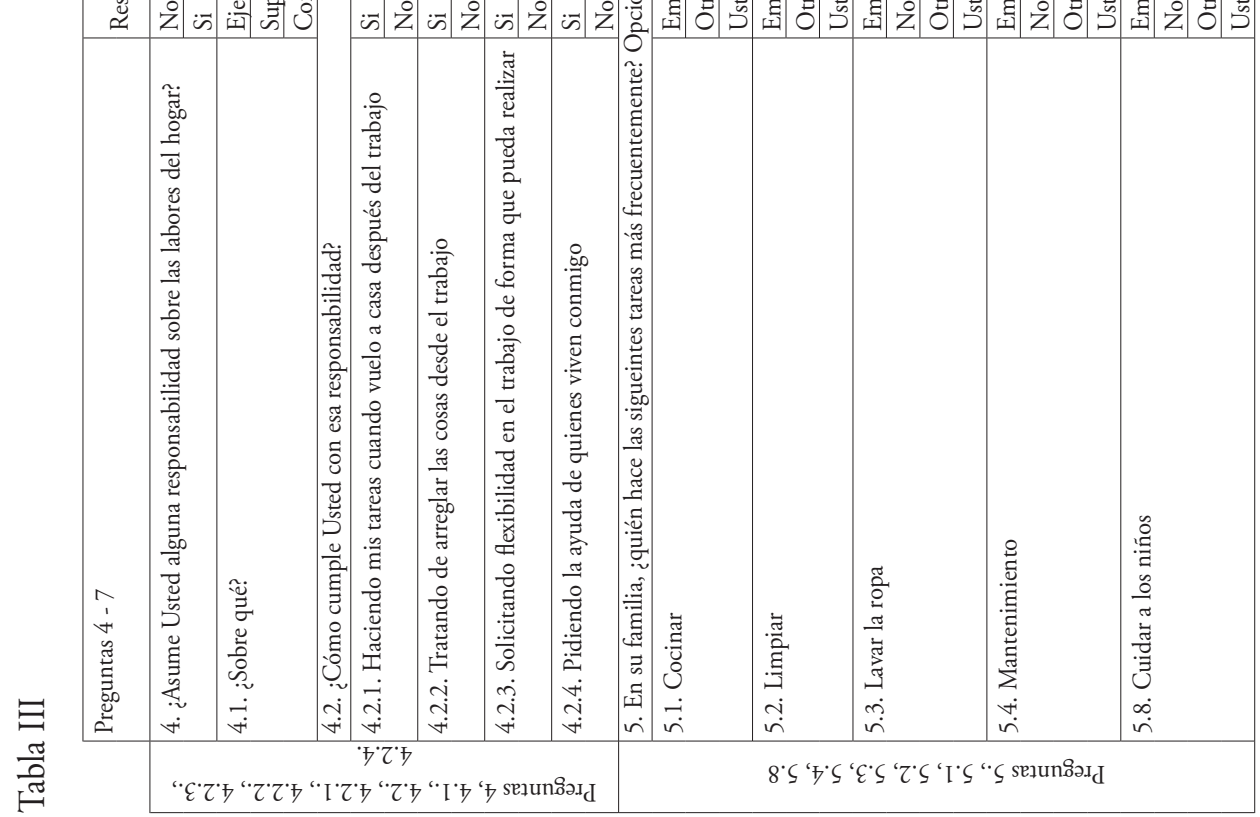




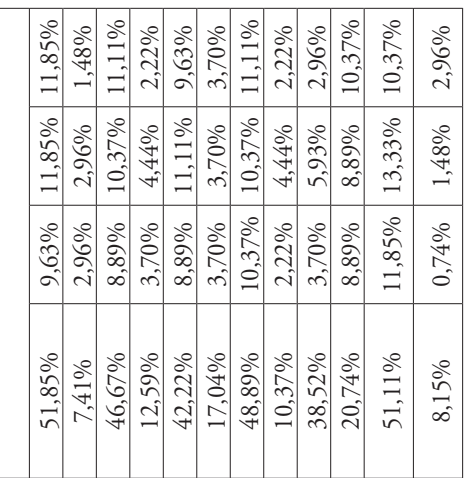

\begin{tabular}{|c|c|c|c|c|c|c|c|c|}
\hline $\begin{array}{l}\text { ते } \\
\text { ते }\end{array}$ & & & & & & & & $\widetilde{\gamma}$ \\
\hline $\begin{array}{l}\text { הें } \\
\text { जे } \\
\text { | }\end{array}$ & 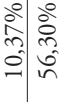 & 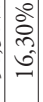 & $\begin{array}{c}0 \\
0 \\
-0\end{array}$ & & & & & 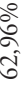 \\
\hline
\end{tabular}

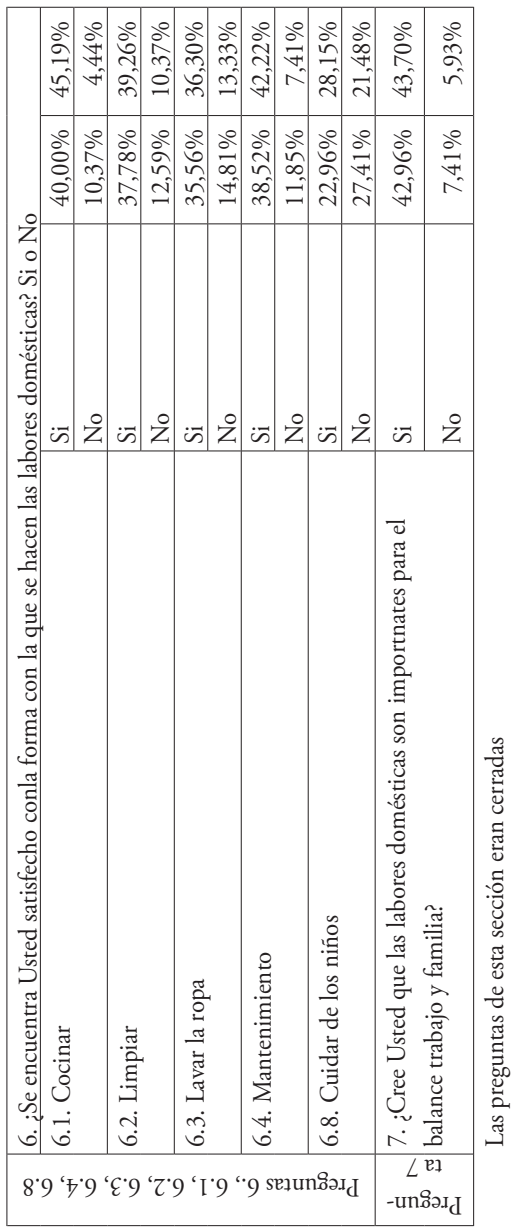


Tabla III A. Con quién viven los hombres que no asumen responsabilidades

\begin{tabular}{|l|l|}
\hline Con quién vive \\
\hline ESH (Con cónyuge e hijos) & $80 \%$ \\
\hline ESP (Con cónyuge) & $10 \%$ \\
\hline SOL (Solo) & $10 \%$ \\
\hline
\end{tabular}

Tabla IV A. Porcentaje de hombres y mujeres que piden o no flexibilidad laboral para cumplir con las responsabilidades del hogar

\begin{tabular}{|l|l|l|}
\cline { 2 - 3 } \multicolumn{1}{c|}{} & Piden flexibilidad & No piden flexibilidad \\
\hline Mujeres & $2,96 \%$ & $47,41 \%$ \\
\hline Hombres & $1,48 \%$ & $48,15 \%$ \\
\hline
\end{tabular}

Tabla IV B. De las mujeres que piden flexibilidad

\begin{tabular}{|l|l|l|l|} 
Estado civil & \multicolumn{2}{|l|}{ Vive con } \\
\hline Casada & $80,00 \%$ & $80,00 \%$ & Esposo e hijos \\
\hline Sola & $20,00 \%$ & $20,00 \%$ & Sola \\
\hline
\end{tabular}

\section{Discusión}

Mirando las dependencias existentes respecto de la pregunta 4-4.2.4 se puede concluir que tan sólo el género se asocia con quién asume la responsabilidad por las labores del hogar. En otras palabras, el acto de asumir responsabilidad por el trabajo del hogar depende del género, donde las mujeres son el grupo que más posiblemente asume al menos alguna responsabilidad sobre el trabajo doméstico. Pero no se encontró ninguna otra relación de importancia para la forma en la cual se cumple con tal responsabilidad. La excepción es la pregunta 4.2.4 que definitivamente está asociada con las personas con quienes vive, toda vez que la pregunta establece que la manera de cumplir con mis responsabilidades es pidiendo la ayuda de aquellos que viven conmigo. Los hallazgos corroboran la literatura sobre la materia, subrayando el hecho de que las mujeres son quienes se acercan a lo doméstico ya sea porque consideran que es lo que se espera de ellas o porque quieren hacerlo o porque están dispuestas a sacrificar su tiempo de familia y/o su tiempo libre para el bienestar de los otros miembros de la casa.

La tabla III A revela que los hombres que no asumen ninguna responsabilidad sobre el trabajo doméstico viven con sus esposas, o con sus esposas e hijos, o con otros, asumiendo que ya que las tareas domésticas deben ser realizadas para asegurar un mínimo de buenas condiciones de vida, alguien más está haciendo el trabajo. Lo que parece sorprendente es que no hay diferencias de importancia en la manera en la que se cumple con la responsabilidad. Sean hombres o mujeres, casados o solteros, que viven solos o con su cónyuge y / o hijos, las responsabilidades del trabajo doméstico usualmente son 
atendidas una vez uno llega a la casa después del trabajo, o tratando de resolver algunos de los problemas involucrados en esa responsabilidad, desde el sitio de trabajo.

Es importante mirar las pocas respuestas positivas dadas a la pregunta 4.2.3 (Tablas IV A, y IV B) que preguntaba que si con el fin de cumplir con la responsabilidad doméstica se había pedido flexibilidad. La mayoría de estas respuestas positivas fueron de mujeres casadas y que vivían con sus maridos e hijos. Las respuestas pueden apuntar una falta de disponibilidad de esta clase de políticas dentro de las empresas colombianas, lo que a su vez sería coherente con los resultados del IFREI 2005. Los resultados de la presente investigación también resaltan la forma en que los colombianos prefieren manejar los asuntos familiares: pidiéndole ayuda a otros miembros de la familia, pertenezcan éstos a la familia inmediata o a la familia extendida (ENV, 2006). Esta tendencia también explicaría el alto número de respuestas positivas para la pregunta 4.2.4. Los datos analizados parcialmente apoyan $\mathrm{H}_{1}$ donde afirmamos que género, estado civil y las personas con quienes se vive están asociados con la forma como se manejaba la responsabilidad doméstica.

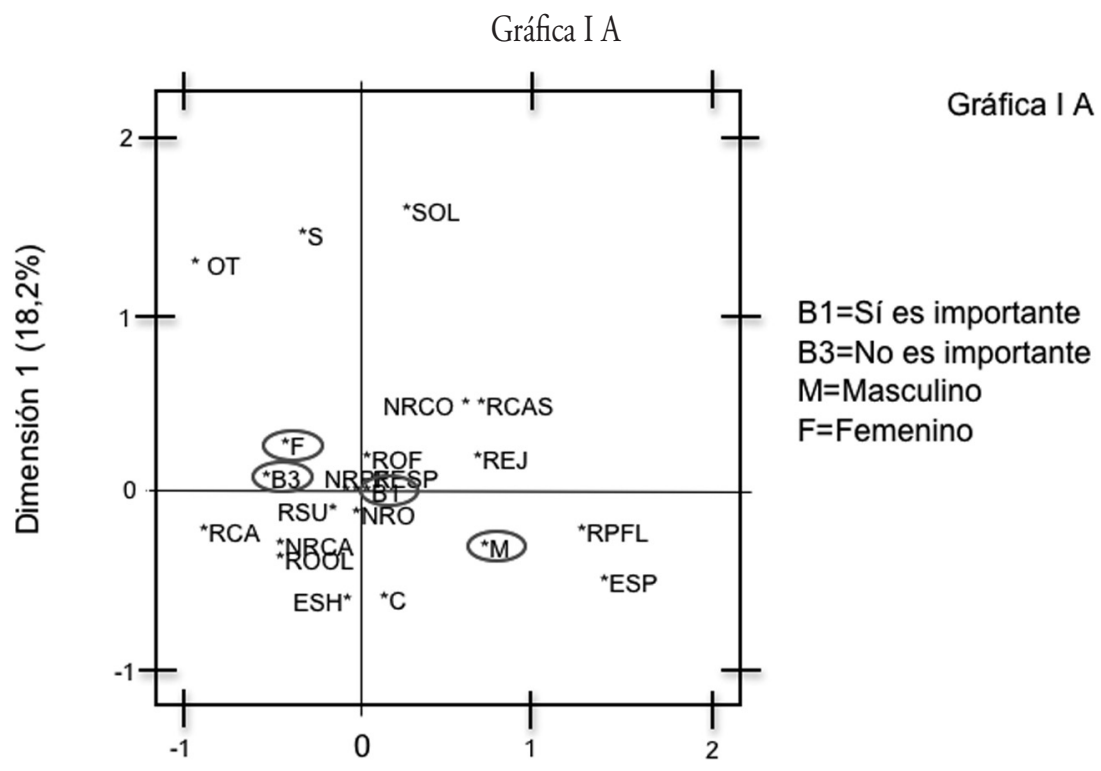

Dimensión $2(14,6 \%)$

La gráfica I A relaciona género, estado civil y personas con quienes se vive con las preguntas 4-4.2.4 y con la 7, donde se pregunta si el trabajo del hogar tiene alguna relevancia en el equilibrio entre trabajo y familia. La alineación de la mujer $(\mathrm{F})$ con B3 en el cuadrante superior izquierdo no apoya nuestra hipótesis de que es más probable que las mujeres (a) que asumen la responsabilidad de las tareas del hogar antes que los hombres (b) quienes también asumen responsabilidades, consideren que el trabajo 
doméstico es importante en el balance entre familia y trabajo. B3 es la codificación para aquellos que respondieron que no consideraban que el trabajo doméstico tuviera alguna relevancia en el balance de la familia y el trabajo. Entre más cerca a la mujer (F), más se identifican los dos grupos. $\mathrm{H} 2$ no tiene soporte.

La segunda parte de las preguntas $(5.1-5.4,5.8)$ se refiere a quién realiza los trabajos del hogar con más frecuencia. El objetivo es determinar quién hace realmente el trabajo. Los datos sugieren que hay importantes dependencias entre la clase de actividad doméstica involucrada y el género y el estado civil. Cocinar, limpiar y el mantenimiento se relacionan con el género, mientras que el estado civil se relaciona con cocinar, limpiar y el cuidado de los niños. Esto quiere decir que el género y el estado civil son buenos indicadores de quién realiza estas tareas con mayor frecuencia. La cocina $(17,04 \%)$ y la limpieza $(10,37 \%)$ son actividades más frecuentemente realizadas por las mujeres; el mantenimiento $(15,56 \%)$ es algo que los hombres hacen más frecuentemente, confirmando así los datos internacionales existentes (Caplan et al., 2006). El estar casado o soltero es algo asociado con quién cocinará y hará la limpieza con mayor frecuencia. Y por supuesto, con quién cuidará los niños. Sin embargo, en los hogares del nivel socioeconómico alto en Colombia, quien cocina, limpia, lava la ropa y hace el mantenimiento con mayor frecuencia, es la empleada del servicio doméstico. Este es un elemento que debe ser considerado cuando la investigación se extienda a otros estratos.

Hay una excepción a este monopolio de servicio doméstico que se acaba de mencionar, y es el caso del cuidado de los nińos. Esta es una actividad que las mujeres hacen más frecuentemente que cualquier otra. En relación con el comportamiento de los hombres como padres, el 12,59\% de los hombres contestaron que ellos cuidan a los niños con mayor frecuencia. Esto definitivamente es un cambio frente a los que ocurría hace 30 años (Wainerman, 2003).

La tercera parte de las preguntas se refiere a la satisfacción con el trabajo doméstico. Los resultados revelan una relación dependiente entre estar satisfecho con el cuidado de los niños y el estado civil y con quién se vive. La relación parece ser consistente con lo que se mencionó respecto de las mujeres y la infelicidad: el estado civil es un elemento importante. Esa situación puede agravarse por la presencia de niños, creando así insatisfacción. En general las mujeres de alguna forma están menos satisfechas que los hombres en las actividades. Nuevamente esto sigue el mismo patrón indicado para la nación entera: en Colombia los hombres parecen ser más felices y estar más satisfechos que las mujeres. Por otro lado, hay una constante del 38\% de los hombres y $38 \%$ de las mujeres que están satisfechos con la forma como se hace el trabajo doméstico. En H4 asumimos que era más probable que una persona que no está satisfecha piense que el trabajo del hogar es relevante en el equilibrio entre trabajo y familia. Una mirada cuidadosa al Gráfico I B nos muestra que este no es el caso. Quienes están insatisfechos con el resultado del trabajo doméstico se ubican en el cuadrante superior derecho, 
exceptuando aquellos insatisfechos con el cuidado de los niños (NCCÑ) quienes se ubican cerca a las mujeres $(\mathrm{F})$ más hacia el centro. B1 y B3 pululan alrededor del centro. Lo que quiere decir que no parece haber relación entre el grado de satisfacción y el considerar el trabajo doméstico como variable importante en el balance familia-trabajo. H4 tampoco tiene soporte.

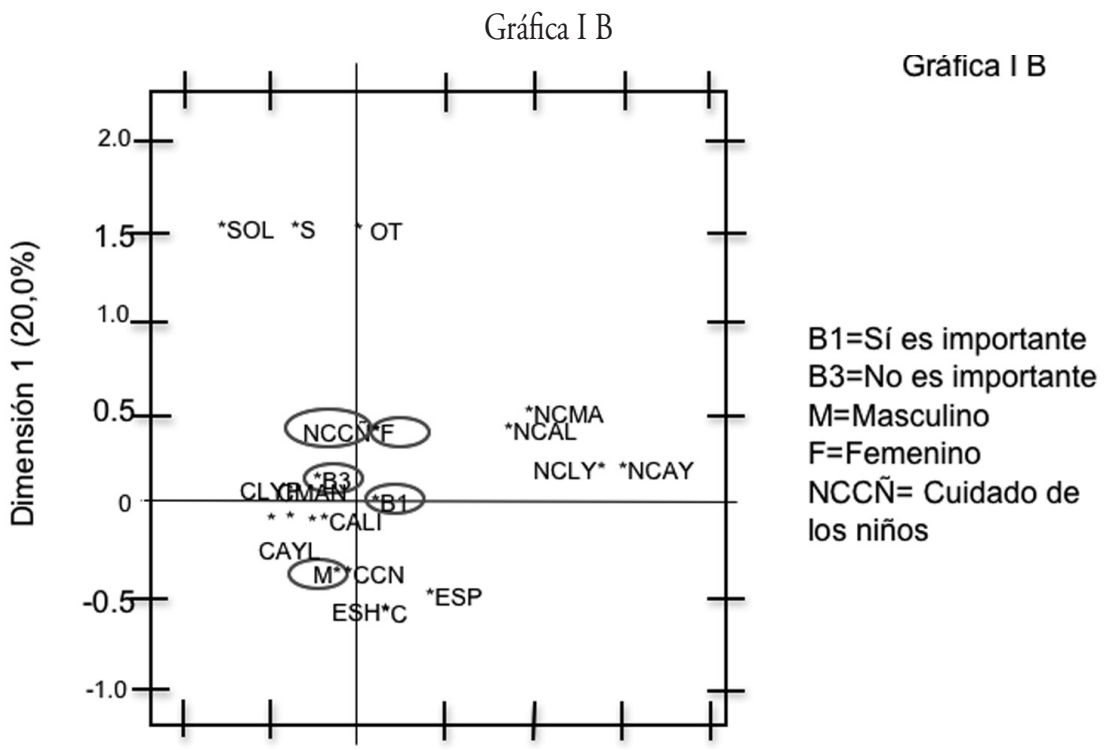

Los resultados para la pregunta 7 (Tabla II) demuestran que los colombianos que viven en Bogotá, de las clases socioeconómicas altas, sin importar el género ni el estado civil, consideran que el trabajo doméstico es relevante en el balance de trabajo y familia. Lo mismo es aplicable para la variable con quién se vive (Tabla III pregunta 6). Así, H3 tampoco está soportada. Y sin embargo el alto porcentaje de aquellos que dijeron que consideraban que el trabajo doméstico era relevante en el equilibrio de trabajo y familia, sin importar el género, el estado civil y las condiciones de vida diaria, muestra un nuevo nivel de conciencia que pronostica un mejor futuro.

\section{Limitaciones y sugerencias}

El presente estudio tiene varias limitaciones. Para empezar la investigación fue exploratoria y pretendía ser descriptiva. Los resultados se pueden generalizar para los colombianos que viven en Bogotá y que pertenecen a los estratos 5 y 6 y sean mayores de 40 ańos, pero no para el resto de colombianos. Es importante proseguir con la investigación en otros estratos y en diferentes regiones, especialmente la costa norte y Antioquia, de modo que se puedan contrastar los resultados y se pueda validar o no las hipótesis en las distintas regiones del país y a los distintos niveles económicos. Así se obtendrá una 
visión más amplia y real. Las preguntas analizadas para este trabajo deben ser puestas en relación con las otras partes de la encuesta y nuevos escenarios pueden aparecer.

El objetivo primario para recolectar datos que nos puedan ayudar a comenzar a pensar en la interfaz familia - trabajo en Colombia se ha conseguido. Sin embargo, surgen nuevas preguntas. La gran aceptación del trabajo doméstico como un elemento relevante para el equilibrio entre trabajo y vida familiar abre nuevas puertas hacia el ámbito doméstico y centra la atención en la permeabilidad de ambos ámbitos: ¿Qué se puede facilitar para permear de un ámbito hacia el otro? ¿Qué mecanismos se pueden desarrollar? ¿Qué se puede hacer para propiciar la transferencia de habilidades y prácticas del uno al otro y viceversa? En la medida en que el esfuerzo involucre a profesionales y a investigadores, empleados y empleadores, el camino puede ser uno de crecimiento para las empresas, las familias y la sociedad en general.

\section{Bibliografía}

Adams, G. A.; King, L. A. \& King, D. W. (1996). "Relationships of job and family involvement, family social support, and work-family conflict with job and life satisfaction". Journal of Applied Psychology, 81: 411-420.

Baltes, B. \& Heydens-Gahir, H. (2003). Reduction of work-family conflict through the use of selection, optimization, and compensation behaviors. Journal of Applied Psychology, 88: 1005-1018.

Barling, J. \& Sorensen, D. (1997). Work and family: In search of a relevant research agenda. En C. L. Cooper \& S. E. Jackson (Eds.), Creating tomorrow's organizations, 157-169. New York: Wiley.

Barnett, R.C. \& Hyde, J.S. (2001). Women, men, work, and family. An expansionist theory. American Psychologist, 56, 781-796.

Batt, R. \& Valcour, P. M. (2003). Human resources practices as predictors of work-family outcomes and employee turnover. Industrial Relations, 42, 189-220.

Blair, S. L. \& Johnson, M. P. (1992). Wives' perceptions of fairness and the division of labor: The intersection of housework and ideology. Journal of Marriage and Family, 54, 570-581.

Bianchi, S. M. ; Milkie, M. M. ; Sayer, L. C. \& Robinson, J. P. (2000). Is anyone doing the housework? Social Forces, 79, 191-228.

Caplan, L. \& Schooler, C. (2006). Household Work Complexity, Intellectual Functioning, and Self-Esteem in Men and Women. Journal of Marriage and Family, 68: 883-900.

Carlson, D. S. \& Kacmar, K. M. (2000). Work-family conflict in the organization: Do life role values make a difference? Journal of Management, 26, 1031-1054. 
Carlson, D. S.; Kacmar, K. M. \& Williams, L. J. (2000). Construction and initial validation of a multidimensional measure of work-family conflict. Journal of Vocational Behavior, 56, 249-276.

Comisión Económica para América Latina. CEPAL. Panorama social de América Latina. Retrieved: November 20, 2005 from http://www.eclac.cl/publicaciones/DesarrolloSocial/O/LCL2220PE/ PSE2004_Cap4_estructuras.pdf.

Coverman, S. (1989). Women's work is never done: The division of domestic labor. En J. Freeman (Ed.), Women: A feminist perspective (4th ed.): 356-367. Mountain View, CA: Mayfield.

Crouter, A. (1984). Spillover from family to work: The neglected side of the workfamily interface. Human Relations, 37: 425-442.

DAPD, Área de Estadística y Monitoreo, 2002.

Departamento Administrativo Nacional de Estadística [DANE] (2005). http://www. dane.gov.co/files/censo2005/gene_15_03_07.pdf. Date of access: June 14, 2007.

Drago, R. \& Kashian, R. (2003). Mapping the terrain of work-family journals. Journal of Family Issues, 24: 488-512.

Duxbury, L.; Higgins, C.; Lee, C. \& Mills, S. (1992). Balancing Work and Family: A Study of the Canadian Public Sector. Prepared for the Department of Health and Welfare Canada (NHRDP), Ottawa.

ENV (2006). Nuestra identidad. Estudio Colombiano de Valores. Bogotá: Raddar.

Ford, M. T.; Heinen, B. A. \& Langkamer, K. L. (2007). Work and family satisfaction and conflict: A meta-analysis of cross-domain relations. Journal of Applied Psychology, 92: 57-80.

Frone, M. R.; Russell, M. \& Cooper, M. L. (1992). Antecedents and outcomes of work-family conflict: Testing a model of the work-family interface. Journal of Applied Psychology, 77, 65-78.

Frone, M. R. (2003). Work-family balance. En J. C. Quick \& L. E. Tetrick (Eds.), Handbook of occupational health psychology, 143-162. Washington, DC: American Psychological Association.

Frone, M. R.; Yardley, J. K. \& Markel, K. S. (1997). Developing and testing an integrative model of the work-family interface. Journal of Vocational Behavior, 50: $145-167$.

Greenberger, E. \& O'Neil, R. 1990. Parents' concerns about their child's development: Implications for fathers' and mothers' well-being and attitudes toward work. Journal of Marriage and Family, 52, 621-635.

Greenhaus, J. H. \& Beutell, N. J. (1985). Sources of conflict between work and family roles. Academy of Management Review, 10, 76-88. 
Greenhaus, J. H. \& Parasuraman, S. (1999). Research on work, family, and gender: Current status and future directions. In G. N. Powell (Ed.), Handbook of gender and work, 391-412. Newbury Park, CA: Sage.

Greenhaus, J. H. \& Powell, G.N. (2006). When work and family are allies: A theory of work-family enrichment. Academy of Management Review, 31,1: 72-92.

Grzywacz, J. G. (2000). Work-family spillover and health during midlife: Is managing conflict everything? American Journal of Health Promotion, 14: 236-243.

Grzywacz, J. G.; Almeida, D. M. \& McDonald, D. A. (2002). Work-family spillover and daily reports of work and family stress in the adult labor force. Family Relations, 51: 28-36.

Grzywacz, J. H. \& Marks, N. F. (2000a). Family, work, work family spillover, and problem drinking during midlife. Journal of Marriage and the Family, 62: 336-348.

Grzywacz, J. G. \& Marks, N. F. (2000b). Reconceptualizing the work-family interface: An ecological perspective on the correlates of positive and negative spillover between work and family. Journal of Occupational Health Psychology, 5: $111-126$.

Gutek, B. A.; Searle, S. \& Klepa, L. (1991). Rational versus gender-role explanations for work-family conflict. Journal of Applied Psychology, 76: 560-568.

Hammer, L. B.; Cullen, J. C.; Caubet, S.; Johnson, J.; Neal, M. B. \& Sinclair, R. R. (2002). The effects of work-family fit on depression: A longitudinal study. Paper presented at the 17 th Annual Meeting of SIOP, Toronto.

Hanson, G. C. ; Colton, C. L. \& Hammer, L. B. (2003). Development and validation of a multidimensional scale of work-family positive spillover. Paper presented at the 18th Annual Meeting of SIOP, Orlando.

Hochschild, A.R. (1999). The managed heart: Commercialization of human feeling. Berkeley, CA: University of California Press.

Idrovo, S. (2006). Las políticas de conciliación trabajo-familia en las empresas colombianas. Estudios Gerenciales, 100: 49-71.

Jansen, N. W. H.; Kant, I.; Kristensen, T. S. \& Nijhuis, F. J. N. (2003). Antecedents and consequences of work-family conflict: A prospective cohort study. Journal of Occupational and Environmental Medicine: 45, 479-491.

Kinnunen, U. \& Gerris, J. (1996). Work experiences and family functioning among employed fathers with children of school age. Family Relations, 45: 449455.

Kirchmeyer, C. (1992). Nonwork participation and work attitudes: A test of scarcity vs. expansion models of personal resources. Human Relations, 45: 775-795.

Kirchmeyer, C. (1992b). Perceptions of nonwork-to-work spillover: Challenging the common view of conflict-ridden domain relationships. Basic and Applied Social Psychology, 13: 231-249. 
Kirchmeyer, C. (1993). Nonwork-to-work spillover: A more balanced view of the experiences and coping of professional women and men. Sex Roles, 28: 531-552.

Kirchmeyer, C. (1995). Managing the work-nonwork boundary: An assessment of organizational responses. Human Relations, 48: 515-536.

Kluwer, E. S. \& Heesink, J. A. M. (1996). Marital conflict about the division of household labor and paid work. Journal of Marriage and Family, 58: 958-969.

León, M. (1989). Domestic Labor and Domestic Service in Colombia. En Chaney, E. M. García Castro, M. (eds.) Muchachas no more. Household workers in Latin America and the Caribbean, 323-349. Philadelphia: Temple University Press.

Lilly, J.D.; Duffy, J. A. \& Virick, M. (2006). A gender-sensitive study of McClelland's needs, stress, and turnover intent with work-family conflict. Women in Management Review, 21: 662-680.

Lingard, H. \& Francis, V. (2007). "Negative interference” between Australian construction professionals' work and family roles. Evidence of an asymmetrical relationship. Engineering, Construction and Architectural Management, 14: 79-93

Maxfield, S. (2005). Mujeres en el Límite. Poder corporativo en América Latina. Report of the Women'S Leadership Conference of the Americas with the collaboration of Inter - American Dialogue and the Simmons Graduate School of Management.

http://www.simmons.edu/som/docs/centers/Al_limite._Edicion_final.pdf. Datos de accesso: June 14, 2007.

Noonan, M.C.; Estes, S.B. \& Glass, J. (2007). Do Workplace Flexibility Policies Influence Time Spent in Domestic Labor? Journal of Family Issues, 28: 263-288.

Pocock, B. (2003). The work/life collision. Sidney: The Federation Press.

Poelmans, S.; O’Driscoll, M. \& Beham, B. (2005). An Overview of International Research on the Work-Family Interface. En Poelmans, S. (ed.), Work and family. An International Research Perspective, 3-46. Mahwah, N.J.: Lawrence Earlbaum Associates.

Rothbard, N. P. (2001). Enriching or depleting? The dynamics of engagement in work and family roles. Administrative Science Quarterly, 46: 655-684.

Rothausen, T.J. (2003). Gender: work-family ideologies and roles. Sloan Work and Family Encyclopedia.

Ruderman, M. N.; Ohlott, P. J.; Panzer, K. \& King, S. N. (2002). Benefits of multiple roles for managerial women. Academy of Management Journal, 45: 369-386. 
Shaffer, M. A.; Francesco, A.M.; Joplin, J.R.W.; Lau, T. (2005). Easing the pain: A Cross-cultural study of support resources and their influence on work-family conflict. En Poelmans, S. (ed.), Work and family. An International Research Perspective, 319-340. Mahwah, N.J.: Lawrence Earlbaum Associates.

Spector, P. E.; Cooper, C. L.; Poelmans, S.; Allen, T. D.; O’Driscoll, M.; Sanchez, J. I. et al. (2004). A cross-national comparative study of work-family stressors, working hours, and well-being: China and Latin America versus the Anglo world. Personnel Psychology, 57, 119-142.

Spector, P.; Miller, K.; Poelmans, S.; Cooper, C.; Berwier, P.; Hart, P.; Lu, L.; Renault de Moraes, L.; Ostrognay, G.; Pitariu, H.; Salamatov, V.; Widerszal-Bazyl, M. \& Yu, S. (2005). An International Comparative Study of Work-family Stress and Occupational Strain. En Poelmans, S. (ed.). Work and family. An International Research Perspective, 71-84. Mahwah, N.J.: Lawrence Earlbaum Associates.

Spitze, G. (1988). Women's employment and family relations: A review. Journal of Marriage and Family, 50, 595-618.

Stephens, M. A. P.; Franks, M. M. \& Atienza, A. A. (1997). Where two roles intersect: Spillover between parent care and employment. Psychology and Aging, 12: 30-37.

Stevens, D. P.; Kiger, G. \& Mannon, S. E. (2005). Domestic labor and marital satisfaction: How much or how satisfied? Marriage \& Family Review, 37, 49-67.

Stevens, D. P.; Kiger, G. \& Riley, P. J. (2001). Working hard or hardly working: Domestic labor and marital satisfaction among dual-earner couples. Journal of Marriage and Family, 63, 514-526.

Stevens, D. P.; Kiger, G. \& Riley, P. J. (2002). Coming unglued? Workplace characteristics, work satisfaction, and family cohesion. Social Behavior and Personality, 30, 289-302.

Stevens, D.P.; Minnotte, K. L.; Mannon, S.E. \& Kiger, G. (2007). Examining the Neglected Side of the Work-Family Interface: Antecedents of Positive and Negative Family-to-Work Spillover. Journal of Family Issues, 28: 242-262.

Sumer, H. C. \& Knight, P. A. (2001). How do people with different attachment styles balance work and family? A personality perspective on work-family linkage. Journal of Applied Psychology, 86: 653-663.

Tausig, M. \& Fenwick, R. (2001). Unbinding Time: Alternate Work Schedules and Work-Life Balance. Journal of Family and Economic Issues, 22:101-119.

Thomas, L. T. \& Ganster, D.C. (1995). Impact of family service-supportive work variables on work-family conflict and strain: a control perspective. Journal of Applied Psychology, 80: 6-15.

Tiedje, L. B.; Wortman, C. B.; Downey, G.; Emmons, C.; Biernat, M. \& Lang, R. (1990). Women with multiple roles: Role-compatibility perceptions, 
satisfaction, and mental health. Journal of Marriage and the Family, 52: 63-72.

Tompson, H. B. \& Werner, J. M. (1997). The impact of role conflict/facilitation on core and discretionary behaviors: Testing a mediated model. Journal of Management, 23: 583-601.

Voydanoff, P. (1988). Work role characteristics, family structure demands, and work/ family conflict. Journal of Marriage and Family, 50, 749-761.

Voydanoff, P. (2001). Incorporating community into work and family research: A review of basic relationships. Human Relations, 54: 1609-1637.

Wainerman, C. (2003). Padres y Maridos. Los varones en la familia. En Wainerman, C. (ed.), Familia, Trabajo y Género. Un mundo de nuevas relaciones: 199224. Buenos Aires: Fondo de Cultura Económica.

Wayne, J. H.; Musisca, N. \& Fleeson, W. (2004). Considering the role of personality in the work-family experience: Relationships of the big five to work-family conflict and facilitation. Journal of Vocational Behavior, 64: 108-130.

Westman, M. (2005). Cross-cultural differences in crossover research. In Poelmans, S. (ed.), Work and family. An International Research Perspective, 241-260. Mahwah, N.J.: Lawrence Earlbaum Associates.

Zimmerman, T. S.; Haddock, S. A.; Current, L. R. \& Ziemba, S. (2003). Intimate partnership: Foundation to the successful balance of family and work. American Journal of Family Therapy, 31, 107-124. 


\section{Apéndice I}

The FREQ Procedure

\begin{tabular}{|c|c|c|c|c|}
\cline { 2 - 5 } \multicolumn{1}{c|}{} & \multicolumn{4}{c|}{ Género } \\
\cline { 2 - 5 } \multicolumn{1}{c|}{ Cumulative } & \multicolumn{2}{c|}{ Cumulative } \\
\hline Género & Frequency & Percent & Frequency & Percent \\
\hline 1 & 68 & $50,37 \%$ & 68 & $50,37 \%$ \\
\hline 2 & 67 & $49,63 \%$ & 135 & $100,00 \%$ \\
\hline
\end{tabular}

\begin{tabular}{|c|c|c|c|c|}
\cline { 2 - 5 } \multicolumn{1}{c|}{} & \multicolumn{4}{c|}{ Living arrangement } \\
\cline { 2 - 5 } \multicolumn{1}{c|}{} & \multicolumn{2}{c|}{ Cumulative } & \multicolumn{2}{c|}{ Cumulative } \\
\hline vive & Frequency & Percent & Frequency & Percent \\
\hline 1 & 18 & $13,33 \%$ & 18 & $13,33 \%$ \\
\hline 2 & 17 & $12,59 \%$ & 35 & $25,93 \%$ \\
\hline 3 & 80 & $59,26 \%$ & 115 & $85,19 \%$ \\
\hline 4 & 10 & $7,41 \%$ & 125 & $92,59 \%$ \\
\hline 5 & 8 & $5,93 \%$ & 133 & $98,52 \%$ \\
\hline 6 & 2 & $1,48 \%$ & 135 & $100,00 \%$ \\
\hline
\end{tabular}

\begin{tabular}{|c|c|c|c|c|}
\cline { 2 - 5 } \multicolumn{1}{c|}{} & \multicolumn{4}{c|}{ Estado civil } \\
\cline { 2 - 5 } \multicolumn{1}{c|}{ Cumulative } & \multicolumn{2}{c|}{ Cumulative } \\
\hline Ecivil & Frequency & Percent & Frequency & Percent \\
\hline 1 & 98 & $72,59 \%$ & 98 & $72,59 \%$ \\
\hline 2 & 37 & $27,41 \%$ & 135 & $100,00 \%$ \\
\hline
\end{tabular}

\begin{tabular}{|c|c|c|c|c|}
\cline { 2 - 5 } \multicolumn{1}{c|}{} & \multicolumn{4}{c|}{ Pregunta Y4 } \\
\cline { 2 - 5 } \multicolumn{1}{c|}{ Cumulative } & \multicolumn{2}{c|}{ Cumulative } \\
\hline Y4 & Frequency & Percent & Frequency & Percent \\
\hline NRES & 11 & $8,15 \%$ & 11 & $8,15 \%$ \\
\hline RESP & 124 & $91,85 \%$ & 135 & $100,00 \%$ \\
\hline
\end{tabular}

\begin{tabular}{|c|c|c|c|c|}
\cline { 2 - 5 } \multicolumn{1}{c|}{} & \multicolumn{4}{c|}{ Pregunta Y41 } \\
\cline { 2 - 5 } \multicolumn{1}{c|}{ Cumulative } & \multicolumn{2}{c|}{ Cumulative } \\
\hline Y41 & Frequency & Percent & Frequency & Percent \\
\hline RCA & 28 & $22,58 \%$ & 28 & $22,58 \%$ \\
\hline REJ & 49 & $39,52 \%$ & 77 & $62,10 \%$ \\
\hline RSU & 47 & $37,90 \%$ & 124 & $100,00 \%$ \\
\hline
\end{tabular}

\begin{tabular}{|c|c|c|c|c|}
\cline { 2 - 5 } \multicolumn{1}{c|}{} & \multicolumn{4}{c|}{ Pregunta Y421 } \\
\cline { 2 - 5 } \multicolumn{1}{c|}{ Cumulative } & \multicolumn{2}{c|}{ Cumulative } \\
\hline Y421 & Frequency & Percent & Frequency & Percent \\
\hline NRCA & 88 & $65,19 \%$ & 88 & $65,19 \%$ \\
\hline RCAS & 47 & $34,81 \%$ & 135 & $100,00 \%$ \\
\hline
\end{tabular}

\begin{tabular}{|c|c|c|c|c|}
\cline { 2 - 5 } \multicolumn{1}{c|}{} & \multicolumn{4}{c|}{ Pregunta Y422 } \\
\cline { 2 - 5 } \multicolumn{1}{c|}{ Cumulative } & \multicolumn{2}{c|}{ Cumulative } \\
\hline Y422 & Frequency & Percent & Frequency & Percent \\
\hline NRO & 103 & $76,30 \%$ & 103 & $76,30 \%$ \\
\hline ROF & 32 & $23,70 \%$ & 135 & $100,00 \%$ \\
\hline
\end{tabular}




\begin{tabular}{|c|c|c|c|c|}
\cline { 2 - 5 } \multicolumn{1}{c|}{} & \multicolumn{4}{c|}{ Pregunta Y423 } \\
\cline { 2 - 5 } \multicolumn{1}{c|}{ Cumulative } & \multicolumn{2}{c|}{ Cumulative } \\
\hline Y423 & Frequency & Percent & Frequency & Percent \\
\hline NRPF & 129 & $95,56 \%$ & 129 & $95,56 \%$ \\
\hline RPFL & 6 & $4,44 \%$ & 135 & $100,00 \%$ \\
\hline
\end{tabular}

\begin{tabular}{|c|c|c|c|c|}
\cline { 2 - 5 } \multicolumn{1}{c|}{} & \multicolumn{4}{c|}{ Pregunta Y424 } \\
\cline { 2 - 5 } \multicolumn{1}{c|}{ Cumulative } & \multicolumn{2}{c|}{ Cumulative } \\
\hline Y424 & Frequency & Percent & Frequency & Percent \\
\hline NRCO & 65 & $48,15 \%$ & 65 & $48,15 \%$ \\
\hline RCOL & 70 & $51,85 \%$ & 135 & $100,00 \%$ \\
\hline
\end{tabular}

\begin{tabular}{|c|c|c|c|c|}
\cline { 2 - 5 } \multicolumn{1}{c|}{} & \multicolumn{4}{c|}{ Pregunta Y51 } \\
\cline { 2 - 5 } \multicolumn{1}{c|}{ Cumulative } & \multicolumn{2}{c|}{ Cumulative } \\
\hline Y51 & Frequency & Percent & Frequency & Percent \\
\hline $\mathrm{AE}$ & 82 & $60,74 \%$ & 82 & $60,74 \%$ \\
\hline $\mathrm{AO}$ & 21 & $15,56 \%$ & 103 & $76,30 \%$ \\
\hline $\mathrm{AU}$ & 32 & $23,70 \%$ & 135 & $100,00 \%$ \\
\hline
\end{tabular}

\begin{tabular}{|c|c|c|c|c|}
\cline { 2 - 5 } \multicolumn{1}{c|}{} & \multicolumn{4}{c|}{ Pregunta Y52 } \\
\cline { 2 - 5 } \multicolumn{1}{c|}{ Cumulative } & \multicolumn{2}{c|}{ Cumulative } \\
\hline Y52 & Frequency & Percent & Frequency & Percent \\
\hline LE & 87 & $64,44 \%$ & 87 & $64,44 \%$ \\
\hline LO & 30 & $22,22 \%$ & 117 & $86,67 \%$ \\
\hline LU & 18 & $13,33 \%$ & 135 & $100,00 \%$ \\
\hline
\end{tabular}

\begin{tabular}{|c|c|c|c|c|}
\cline { 2 - 5 } \multicolumn{1}{c|}{} & \multicolumn{4}{c|}{ Pregunta Y53 } \\
\cline { 2 - 5 } \multicolumn{1}{c|}{ Cumulative } & \multicolumn{2}{c|}{ Cumulative } \\
\hline Y53 & Frequency & Percent & Frequency & Percent \\
\hline PE & 83 & $61,48 \%$ & 83 & $61,48 \%$ \\
\hline PN & 2 & $1,48 \%$ & 85 & $62,96 \%$ \\
\hline PO & 34 & $25,19 \%$ & 119 & $88,15 \%$ \\
\hline PU & 16 & $11,85 \%$ & 135 & $100,00 \%$ \\
\hline
\end{tabular}

\begin{tabular}{|c|c|c|c|c|}
\cline { 2 - 5 } \multicolumn{1}{c|}{} & \multicolumn{4}{c|}{ Pregunta Y54 } \\
\cline { 2 - 5 } \multicolumn{1}{c|}{ Cumulative } & \multicolumn{2}{c|}{ Cumulative } \\
\hline Y54 & Frequency & Percent & Frequency & Percent \\
\hline ME & 83 & $61,48 \%$ & 83 & $61,48 \%$ \\
\hline MN & 5 & $3,70 \%$ & 88 & $65,19 \%$ \\
\hline MO & 16 & $11,85 \%$ & 104 & $77,04 \%$ \\
\hline MU & 31 & $22,96 \%$ & 135 & $100,00 \%$ \\
\hline
\end{tabular}

\begin{tabular}{|c|c|c|c|c|}
\cline { 2 - 5 } \multicolumn{1}{c|}{} & \multicolumn{4}{c|}{ Pregunta Y58 } \\
\cline { 2 - 5 } \multicolumn{1}{c|}{ Cumulative } & \multicolumn{2}{c|}{ Cumulative } \\
\hline Y58 & Frequency & Percent & Frequency & Percent \\
\hline CE & 37 & $27,41 \%$ & 37 & $27,41 \%$ \\
\hline CN & 51 & $37,78 \%$ & 88 & $65,19 \%$ \\
\hline CO & 12 & $8,89 \%$ & 100 & $74,07 \%$ \\
\hline CU & 35 & $25,93 \%$ & 135 & $100,00 \%$ \\
\hline
\end{tabular}




\begin{tabular}{|c|c|c|c|c|}
\cline { 2 - 5 } \multicolumn{1}{c|}{} & \multicolumn{4}{c|}{ Pregunta Y61 } \\
\cline { 2 - 5 } \multicolumn{1}{c|}{ Cumulative } & \multicolumn{2}{c|}{ Cumulative } \\
\hline Y61 & Frequency & Percent & Frequency & Percent \\
\hline CALI & 115 & $85,19 \%$ & 115 & $85,19 \%$ \\
\hline VCAL & 20 & $14,81 \%$ & 135 & $100,00 \%$ \\
\hline
\end{tabular}

\begin{tabular}{|c|c|c|c|c|}
\cline { 2 - 5 } \multicolumn{1}{c|}{} & \multicolumn{4}{c|}{ Pregunta Y62 } \\
\cline { 2 - 5 } \multicolumn{1}{c|}{ Cumulative } & \multicolumn{2}{c|}{ Cumulative } \\
\hline Y62 & Frequency & Percent & Frequency & Percent \\
\hline CAYL & 104 & $77,04 \%$ & 104 & $77,04 \%$ \\
\hline NCAY & 31 & $22,96 \%$ & 135 & $100,00 \%$ \\
\hline
\end{tabular}

\begin{tabular}{|c|c|c|c|c|}
\cline { 2 - 5 } \multicolumn{1}{c|}{} & \multicolumn{4}{c|}{ Pregunta Y63 } \\
\cline { 2 - 5 } \multicolumn{1}{c|}{ Cumulative } & \multicolumn{2}{c|}{ Cumulative } \\
\hline Y63 & Frequency & Percent & Frequency & Percent \\
\hline CLYP & 97 & $71,85 \%$ & 97 & $71,85 \%$ \\
\hline NCLY & 38 & $28,15 \%$ & 135 & $100,00 \%$ \\
\hline
\end{tabular}

\begin{tabular}{|c|c|c|c|c|}
\cline { 2 - 5 } \multicolumn{1}{c|}{} & \multicolumn{4}{c|}{ Pregunta Y64 } \\
\cline { 2 - 5 } \multicolumn{1}{c|}{ Cumulative } & \multicolumn{2}{c|}{ Cumulative } \\
\hline Y64 & Frequency & Percent & Frequency & Percent \\
\hline CMAN & 109 & $80,74 \%$ & 109 & $80,74 \%$ \\
\hline NCMA & 26 & $19,26 \%$ & 135 & $100,00 \%$ \\
\hline
\end{tabular}

\begin{tabular}{|c|c|c|c|c|}
\cline { 2 - 5 } \multicolumn{1}{c|}{} & \multicolumn{4}{c|}{ Pregunta Y68 } \\
\cline { 2 - 5 } \multicolumn{1}{c|}{ Cumulative } & \multicolumn{2}{c|}{ Cumulative } \\
\hline Y68 & Frequency & Percent & Frequency & Percent \\
\hline CCNN & 69 & $51,11 \%$ & 69 & $51,11 \%$ \\
\hline NCCN & 66 & $48,89 \%$ & 135 & $100,00 \%$ \\
\hline
\end{tabular}

\begin{tabular}{|c|c|c|c|c|}
\cline { 2 - 5 } \multicolumn{1}{c|}{} & \multicolumn{4}{c|}{ Pregunta Y7 } \\
\cline { 2 - 5 } \multicolumn{1}{c|}{ Cumulative } & \multicolumn{2}{c|}{ Cumulative } \\
\hline Y7 & Frequency & Percent & Frequency & Percent \\
\hline B1 & 117 & $86,67 \%$ & 117 & $86,67 \%$ \\
\hline B2 & 18 & $13,33 \%$ & 135 & $100,00 \%$ \\
\hline
\end{tabular}

\section{Cómo citar este artículo:}

Hernáez G., Milagrosa e Idrovo C., Sandra (2010). "Armonizando trabajo y familia en Bogotá-Colombia: la conexión doméstica”, Oikos No 29, 65 - 90, Escuela de Administración y Economía, Universidad Católica Silva Henríquez (UCSH), Santiago de Chile. [http://edicionesucsh.cl/oikos/]

Fecha de recepción: 16 / 04 / 2010

Fecha de aprobación: 08/ $10 / 2010$ 